\title{
Fixed pigmented erythema due to cimetidine
}

\section{Almamy Diabaté1, Romain Boussou Aka', Bamba Vagamon', Hamdan Sarah Kourouma², Irenée Gué1, Mamadou Kaloga²}

${ }^{1}$ Department of Dermatology, University Teaching Hospital, Bouake, Côte d'Ivoire, ${ }^{2}$ Department of Dermatology, University Teaching Hospital of Treichville, Abidjan, Côte d'Ivoire

Corresponding author: Dr. Almamy Diabaté, E-mail: docalmamy@yahoo.fr

\begin{abstract}
Fixed pigmented erythema (EPF) is pathognomonic of drug-induced toxicity. Antisecretory drugs are among the most commonly not responsible drugs. EPF secondary to cimetidine administration has rarely been described. We report an EPF case during a cimetidine-based treatment in Bouake. A 43-year-old patient, HIV negative, consults for an EPF located on both sides of the breasts, on the $5^{\text {th }}$ day of an antiulcer treatment comprising compressed cimetidine and gastric dressing based on phosphatase d 'aluminum. The diagnosis is made in front of the characteristic aspect of the lesion of EPF and the early reappearance of lesions recurring exactly in the same places, after the reintroduction of cimetidine. The discovery of an EPF imposes a strict etiological approach in search of the responsible drug. During anti-ulcer treatment, the practitioner should bear in mind the potential role of cimetidine.
\end{abstract}

Key words: Cimetidine; Fixed pigmented erythema; Bouaké (RCI)

\section{INTRODUCTION}

The drug toxidermias are the skin side effects of drugs [1]. These toxidermias may display in the particular aspect as a fixed pigmented erythema (FPE). The most commonly used drugs, including barbiturates, carbamazepine, paracetamol, phenazone, and other pyrazole derivatives [2], might induce a lonely $[3,4]$ or a polymorphic FPE with diverse causes [5-8]. We described a case observed at the University Teaching Hospital of Bouake.

\section{CASE REPORT}

A 43-year-old man, with no medical background and HIV negative, consulted at the Dermatology Department at the University Teaching Hospital of Bouake for an itching pigmented macule which occurred five days after taking cimetidine. The patient consulted for an epigastralgia five days earlier during a medical consultation sponsored by the Member of Parliament of his region and received free care of cimetidine ( 1 tablet of $300 \mathrm{mg}$ per day). On the fifth day of treatment, the patient had chest itching and burning sensation. The physical examination showed several inflammatory and pigmented erythemas on the thorax at the top and bottom of the right and left breasts, which indicated the diagnosis of FPE (Figs. la and lb). The relief of symptoms occurred on the seventh day when he stopped using the drug. However, the subsequent administration of cimetidine resulted in the reappearance of the initial symptoms. As a result, the final withdrawal of cimetidine led to the complete relief of signs. The treatment of the patient was extended for a further four weeks with omeprazole without any incident.

\section{DISCUSSION}

The FPE is the exclusive dermatosis caused by drugs. The most commonly used drugs are some anticonvulsants (barbiturate and carbamazepine), phenazone, acyclovir, hydroxyzine, non steroidal antiinflammatory drugs [1] and certain antibiotics (cyclins, sulphonamides, ampicillin, antituberculosis drugs) [2].

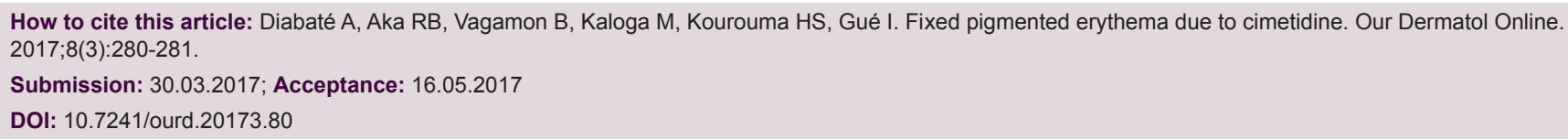




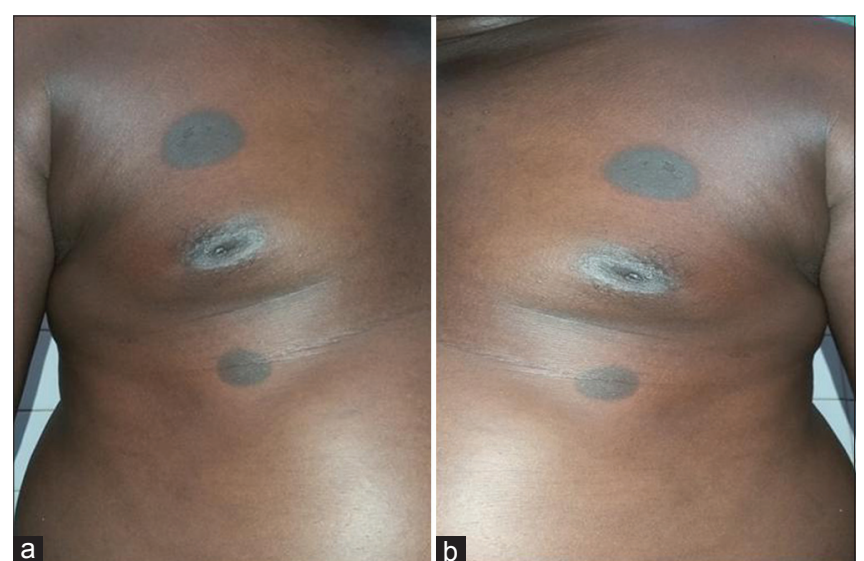

Figure 1: $(a$ and $b)$ Pigmented erythema fixed at the top below the right and left breasts.

Cimetidine was initially verified to induce the FPE in Tokyo in 1985 [3] and in California in 1998 [4]. The leading role of cimetidine in the occurrence of the FPE seems to be certain within our patient since it is the unique systemic drug taken. In addition, dietary risk factors are rarely responsible for FPE even though our patient's eating habits were unchanged. The regression of the symptoms after stopping the use of cimetidine followed by early reappearance ( $<24$ hours) of these initial signs due to the experimental administration of the drug strongly confirm the diagnosis certainty of FPE. In this case, the lesions appeared on the fifth day of the antisecretory treatment. Generally, the time to onset of the FPE is very short (24-48 h) [2] and much shorter in the case of reintroduction of the drug, which is similar to that of our patient.

\section{CONCLUSION}

The diagnosis of FPE requires a rigorous etiological approach. Besides the most frequently used drugs, cimetidine can be responsible for FPE. Therefore, the practitioner should initially consider the potential role of cimetidine.

\section{REFERENCES}

1. Roujeau JC, Bonnetblanc JM, Schmutz JL, Crickx B. Iatro-génie. Diagnostic et prévention. Ann Dermatol Venereol. 2002;129:S163-9.

2. Brahimi N, Routier E, Raison-Peyron N, Tronquoy AF, PougetJasson C, Amarger S, et al. A three-year analysis of FDE inhospital settings in France. Eur J Dermatol. 2010;20:461-4.

3. Helmbold P, Hegemann B, Dickert C, Marsch WC. Symmetric ptychotropic and non pigmenting fixed drug eruption due to cimetidine (so-called baboon syndrome). Dermatology. 1998;197:402-3.

4. Inoue A, Teramae H, Hisa T, Taniguchi S, Chanoki M, Hamada T. Fixed drug eruption due to cimetidine. Acta Derm Venereol. 1995; 75:250.

5. Thyagarajan K, Kamalam A, Thambiah AS. Fixed drug eruptionto griseofulvin. Mykosen. 1981;24:482-4.

6. Boudghene-Stambouli O, Mérad-Boudia A. Fixed drug eruption induced by griseofulvin. Dermatologica. 1989;179:92-3.

7. Mahboob A, Haroon TS. Drugs causing fixed eruptions: a study of450 cases. Int J Dermatol. 1998;37:833-8.

8. Rahman MH. Fixed drug eruption in Bangladeshi population: confirmed by provocative test. Int J Dermatol. 2014;53:255-8.

Copyright by Almamy Diabaté, et al. This is an open access article distributed under the terms of the Creative Commons Attribution License, which permits unrestricted use, distribution, and reproduction in any medium, provided the original author and source are credited.

Source of Support: Nil, Conflict of Interest: None declared. 